\title{
Rigor is Needed when Making Comparative Analysis of Biologics in Severe Asthma
}

Charlene M Prazma, $\mathrm{PhD}^{1}$, Rohit Katial, $\mathrm{MD}^{2}$, Peter Howarth, MD. ${ }^{3}$, Eric Bradford, $\mathrm{MD}^{4}$, Neil Martin, $\mathrm{MD}^{3}$, Emilio Pizzichini, $\mathrm{MD}^{3}$.

${ }^{1}$ Respiratory Medical Franchise, GSK, Research Triangle Park, NC, USA

${ }^{2}$ US Medical Affairs, GSK, Research Triangle Park, NC, USA

${ }^{3}$ Respiratory Medical Franchise, GSK, Brentford, UK.

${ }^{4}$ Respiratory Therapeutic Area, GSK, Research Triangle Park, NC, USA

\section{Corresponding Author:}

Name: Charlene M Prazma, PhD

Address: Research Triangle Park, 5 Moore Drive, PO Box 13398, North Carolina 27709

Tel: +1 919 483-9013

Email: charlene.m.prazma@gsk.com

Words: $970 / 1000(\max )$

References: $11 / 10$ (max)

Tables/Figures: $1 / 2$ (max) 
To the Editor:

We read with caution the study described by Mukherjee and colleagues reporting observations from a 24-week single-blind study of 10 prednisone-dependent patients (1). The described study is nonrandomized and retrospectively compares intravenous reslizumab treatment to subcutaneous mepolizumab treatment in a subset of patients from the previously reported COSMOS study (2), described by the authors as phase 1 . These elements of study design and population require further clarification as they have implications on the robustness of the conclusions of the paper.

Patients included in the study by Mukherjee et al were from one center of the multi-center 52-week open-label extension COSMOS study. COSMOS enrolled patients directly from the 24-week double-blind (mepolizumab vs placebo), randomized, placebo-controlled, SIRIUS steroid sparing study (3). Thirteen patients from SIRIUS (mepolizumab $=8$, placebo $=5$ ) entered COSMOS (phase 1 ) from this center. The demographics and clinical outcomes for this sub-population have been previously reported by Sehmi et al (4) and demonstrated that mepolizumab treated patients responded with a reduction in blood eosinophils, had a trend to sputum eosinophil reduction, a reduction in maintenance OCS dose, and an improvement in ACQ score as compared to placebo. In the study described by the authors this subpopulation was without mepolizumab treatment for at least a 14-month period during which time they were seen in clinical practice and re-established on optimized maintenance OCS dose, following which 10 of the 13 patients enrolled into the interventional phase of this study (phase 2). The understanding of the patient journey through the various studies is particularly relevant as the patient demographics and pre-mepolizumab baseline data reflect data summarized from a mixed population of mepolizumab and placebo treatment patients. When comparing the pre/post mepolizumab data for the primary outcomes of blood and sputum eosinophils this translates into less room for improvement as compared to pre/post reslizumab. From the data presented approximately half of the pre-mepolizumab population from COSMOS had a sputum count below the 3\% threshold at baseline, and 4 of 10 had a blood eosinophil count $<300$ cells $/ \mu$, likely representing the mepolizumab treated patients from SIRIUS. In contrast, the pre-reslizumab baseline data were collected following a 12-month period during which all patients were without mepolizumab treatment, following which, and not unexpectedly (5), all patients had a sputum eosinophil count $>3 \%$ and blood eosinophil count $\geq 300$ cells. These baseline differences make it impossible to draw the conclusion that the magnitude of treatment effect is greater with reslizumab compared to mepolizumab. 
With respect to other efficacy conclusions, the authors describe criteria to define responders and nonresponders, which include a comparison to baseline for the reduction in blood and sputum eosinophils which is not a convincing comparison for the reasons described above. In addition, the authors also inappropriately included occurrence of exacerbations as part of the criteria, comparing the exacerbation rate from a 52-week study to a 16-week study in a sample of 10 patients. The safety data presented should also be put into context of the patient journey described; i.e. the total duration of mepolizumab treatment exposure was 52-76 weeks while compared here to 16 weeks of reslizumab exposure.

The clinical program that supported mepolizumab regulatory approval in 2015 included over 1000 patients who participated in randomized, controlled trials, across multiple countries and centres. This program found that mepolizumab, administered intravenously (DREAM) and in latter studies subcutaneously, led to significant and consistent exacerbation rate reduction, and identified the blood eosinophil count as an effective biomarker to indicate clinical response $(3,6-7)$. In contrast the sputum data from a subset in DREAM did not identify reduction in percent sputum eosinophil as a determinant of exacerbation reduction, irrespective of the baseline level ( $\geq$ or $<3 \%$ ) (6). Post-hoc analysis showed a pharmacodynamic effect with a 78\%, $90 \%$ and $93 \%$ reduction from baseline in sputum eosinophil percent with the $75 \mathrm{mg}, 250 \mathrm{mg}$ and $750 \mathrm{mg}$ IV doses, respectively, compared to a $5 \%$ increase with placebo by 4 weeks of treatment, in those who entered DREAM with a baseline of $\geq 3 \%$ eosinophils (Table 1). With respect to mepolizumab clinical effect in the OCS dependent population, a post-hoc analysis from DREAM showed a similar clinical response as measured by exacerbation rate reduction in the OCS and non-OCS dependent populations $(6,8)$. When studied prospectively (SIRIUS; $n=135)$ in the OCS-dependent population mepolizumab (100mg SC) led to an OCS dose reduction that was consistent, when adjusted for placebo, with the results from the prior study of higher dose mepolizumab (750 mg IV) conducted by Nair et al (9) and also consistent, when adjusted for placebo, with $50 \%$ OCS reduction seen in the more recent benralizumab ZONDA study (10). Additionally, in the sputum sub-study of SIRIUS subjects $(n=13)$ demonstrated a trend toward \% sputum eosinophil reduction in a population who achieved OCS reduction and improvement in asthma control as measured by ACQ (4). While it is acknowledged that further understanding of the drivers of eosinophil mediated disease in the OCSdependent population is required, mepolizumab clinical data to date have proven the utility of the blood eosinophil as the biomarker predictive of treatment response in all studies since DREAM and in a more recent meta-analysis (11). 
The work by Mukherjee et al provides interesting mechanistic information about the activation and recruitment of airways eosinophils in the prednisone-dependent population but to use this nonrandomized, retrospective study to make a conclusive statement that one clinical therapy is superior in efficacy or safety to another is both factually incorrect and scientifically misleading. This trial $(n=10)$ should not be over-interpreted when placed alongside extensive randomized controlled trials of anti-IL5 therapies. In the era of personalized medicine it is clear that not all medicines work to the same degree of effectiveness in all patients but further research, in properly designed studies, should lead to more refined phenotyping and understanding of those who might be responding best to different therapeutic options.

\section{Acknowledgements}

DREAM (NCT01000506), SIRIUS (NCT01691508), and COSMOS (NCT01842607) studies were sponsored by GlaxoSmithKline. All authors are GSK employees and shareholders. 


\section{References:}

1. Mukherjee, M., Paramo, F., Kjarsgaard, M., Salter, B., Nair, G., Vigne, N., Radford, K., Sehmi, R., Nair, P. Weight-adjusted intravenous reslizumab in severe asthma with inadequate response to fixed-dose subcutaneous mepolizumab. Am J Resp Crit Care Med 2018; 197(1):38-46.

2. Lugogo, N., Domingo, C. Chanez, P., Leigh, R., Gilson, M., Price, R., Yancey, S., Ortega, H. Longterm Efficacy and Safety of Mepolizumab in Patients With Severe Eosinophilic Asthma: A Multicenter, Open-label, Phase IIIb Study. Clin Ther. 2016; 38 (9): 2058-2070

3. Bel, E., Wenzel, S., Thompson, P., Prazma, C., Keene, O., Yancey, S., Ortega, H., Pavord, I. Oral glucocorticoid-sparing effect of mepolizumab in eosinophilic asthma. N Engl J Med 2014; 371 (13);1189-1197

4. Sehmi, R., Smith, S., Kjarsgaard, M., Radford, K., Boulet, L., Lemiere, C., Prazma, C., Ortega, H., Martin, J., Nair, P. Role of local eosinophilopoietic processes in the development of airway eosinophilia in prednisone-dependent severe asthma. Clin Exp Allergy 2016; 46(6):793-802

5. Halder, P., Brightling, C., Singapuri, A., Haragadon, B., Gupta, S., Monteiro, W., Bradding, P., Green, R., Wardlaw, A., Ortega, H., and Pavord, I. Outcomes after cessation of mepolizumab therapy in severe eosinophilic asthma: A 12-month follow-up analysis. J. Allergy Clin Immunol 2014; 133 (3):921-923

6. Pavord, I., Korn S, Howarth P, Bleecker E, Buhl R, Keene ON, Ortega H, Chanez P. Mepolizumab for severe eosinophilic asthma (DREAM): a multicenter, double-blind, placebo-controlled trial Lancet 2012; 380 (9842); 651-658

7. Ortega, H., Liu, M., Pavord, I., Brusselle, G., FitzGerald, J., Chetta, A., Humbert, M., Katz, L., Keene, O., Yancey, S., Chanez, P., Mepolizumab treatment in patients with severe eosinophilic asthma. N Engl J Med 2014; 371 (13);1198-1207

8. Prazma C., Wenzel, S., Barnes, N., Douglass, J., Hartley, B., Ortega, H. Characterisation of an OCS-dependent severe asthma population treated with mepolizumab. Thorax 2014; 69 (12):1141-1142

9. Nair, P., Pizzichini, M., Kjarsgaard, M., Inman, MD., Efthimiadis, A., Pizzichini, E., Hargreave, F., O’Byrne, P. Mepolizumab for prednisone-dependent asthma with sputum eosinophilia. N Engl J Med 2009; 5:360 (10); 985-993

10. Nair, P., Wenzel, S., Klaus, R., Bourdin, A., Lugogo, N., Kuna, P., Barker, P., Sproule, S., Ponnarambil, S., Goldman, M. Oral Glucocorticoid-Sparing Effect of Benralizumab in Severe Asthma. N Engl J Med 2017; 376 (25); 2448-2458

11. Ortega, H., Yancey, S., Mayer, B., Gunsoy, N., Keene, O., Bleecker, E., Brightling, C., Pavord, I. Severe eosinophilic asthma treated with mepolizumab stratified by baseline eosinophil thresholds; a secondary analysis of the DREAM and MENSA studies. Lancet Resp Med. 2016; 4: 549-556 
Table 1: DREAM sputum sub-group eosinophil reduction with mepolizumab

\begin{tabular}{|c|c|c|c|c|}
\hline & $\begin{array}{c}\text { Placebo } \\
N=27\end{array}$ & $\begin{array}{c}\text { Mepolizumab } \\
\text { 75mg IV } \\
\mathrm{N}=\mathbf{2 0}\end{array}$ & $\begin{array}{c}\text { Mepolizumab } \\
\text { 250mg IV } \\
\mathrm{N}=\mathbf{2 4}\end{array}$ & $\begin{array}{c}\text { Mepolizumab } \\
\text { 750mg IV } \\
\mathrm{N}=\mathbf{2 3}\end{array}$ \\
\hline \multicolumn{5}{|l|}{ Baseline } \\
\hline $\begin{array}{l}\text { Sputum eosinophils } \\
\geq 3 \% \text { (n) }\end{array}$ & 16 & 15 & 20 & 15 \\
\hline $\begin{array}{l}\text { Sputum eosinophils } \\
(\%)^{*}\end{array}$ & $22.28(0.980)$ & $23.55(0.868)$ & $14.58(0.938)$ & $19.20(0.838)$ \\
\hline \multicolumn{5}{|l|}{ Week 4} \\
\hline $\begin{array}{l}\text { Sputum eosinophils } \\
\geq 3 \% \text { ( } n)\end{array}$ & 12 & 9 & 17 & 7 \\
\hline $\begin{array}{l}\text { Sputum eosinophils } \\
(\%)^{*}\end{array}$ & $18.72(1.464)$ & $5.19(2.174)$ & $1.47(1.794)$ & $0.74(1.717)$ \\
\hline Ratio to baseline* & $1.05(0.818)$ & $0.22(1.561)$ & $0.10(1.849)$ & $0.07(1.654)$ \\
\hline
\end{tabular}

*Geometric mean (SD logs), data were log transformed prior to analysis 